https://helda.helsinki.fi

Lower urinary tract symptoms and sexual functions after endorectal pull-through for Hirschsprung disease : controlled long-term outcomes

Neuvonen, Malla

2017-08

Neuvonen, M , Kyrklund, K, Taskinen , S , Koivusalo, A, Rintala , R J \& Pakarinen , M P 2017 , ' Lower urinary tract symptoms and sexual functions after endorectal pull-through for Hirschsprung disease : controlled long-term outcomes ', Journal of Pediatric Surgery, vol. 52 , no. 8 , pp. 1296-1301 . https://doi.org/10.1016/j.jpedsurg.2017.02.013

http://hdl.handle.net/10138/297847

https://doi.org/10.1016/j.jpedsurg.2017.02.013

publishedVersion

Downloaded from Helda, University of Helsinki institutional repository.

This is an electronic reprint of the original article.

This reprint may differ from the original in pagination and typographic detail.

Please cite the original version. 


\title{
Lower urinary tract symptoms and sexual functions after endorectal pull-through for Hirschsprung disease: controlled long-term outcomes ${ }^{2 \pi}$, 访访, $\star$
}

\author{
Malla Neuvonen *, Kristiina Kyrklund, Seppo Taskinen, Antti Koivusalo, Risto J. Rintala, Mikko P. Pakarinen \\ Department of Pediatric Surgery, Children's Hospital, Helsinki University Central Hospital, University of Helsinki, Finland
}

\section{A R T I C L E I N F O}

\section{Article history:}

Received 25 August 2016

Received in revised form 16 February 2017

Accepted 28 February 2017

\section{Key words:}

LUTS

Sexual function

Hirschsprung's disease

Endorectal pull-through

\begin{abstract}
A B S T R A C T
Background/purpose: To define the prevalence of lower urinary tract symptoms (LUTS) and outcomes for sexual function after endorectal pull-through (EPT) for Hirschsprung disease (HD) compared to controls. To date, similar controlled studies are lacking.

Methods: Patients aged $\geq 4$ years $(n=123)$ operated on for HD at our center between 1987 and 2011 were invited to answer questionnaires on LUTS and sexual function (aged $\geq 16$ years). Patients with an intellectual disability and patients with a definitive endostomy were excluded. Patients were matched to three controls and also invited to a clinical follow-up for urological investigations including urine flow measurement, renal tract ultrasound, and urinalysis.

Results: Altogether, 59 responses concerning LUTS and 24 responses concerning sexual functions were analyzed. No significant differences were demonstrated in the overall prevalence of LUTS between patients (67\%) and controls ( $80 \%$ ), nor in the prevalence of frequent LUTS (14\% vs. $16 \%$; $P=$ NS for both). One patient (2\%) had a urethral stricture after laparotomy-assisted EPT. Male patients reported sexual satisfaction and erectile function similar to controls $(P>0.10)$. Female patients were currently less in stable relationships compared to controls ( $25 \%$ vs. $83 \%, P=0.005)$.

Conclusions: Our results support the safety of EPT in patients with HD with regard to preservation of the integrity and functioning of the genitourinary tract.
\end{abstract}

(c) 2017 Elsevier Inc. All rights reserved.
Hirschsprung's disease (HD) is a congenital condition affecting 1:5000 live births characterized by an absence of ganglion cells in the myenteric and submucosal plexuses of the intestine. Surgical treatment involves resection of the distal aganglionic bowel segment and pullthrough of the remaining ganglionic bowel down to the anus [1]. During the last decades, endorectal pull-through (EPT) techniques, including totally transanal operations have become increasingly popular approaches for the management of HD [1-5]. Historically, the original Swenson, Soave and Duhamel operations involved the entire rectal dissection via abdomen. Inadvertent injuries to the genitourinary tract or its innervation may have occasionally occurred during pelvic dissection especially outside the rectal wall in the Swenson and Duhamel operation, resulting in lower urinary tract symptoms (LUTS) and sexual

\footnotetext{
it Conflicts of interest: None to declare.

is 2 Sources of funding: This research was supported by grants from the Finnish Pediatric Research Foundation, Päivikki and Sakari Sohlberg Foundation, the Helsinki University Central Hospital research funds and the Sigrid Juselius Foundation.

$\star$ Reprints: Reprints will not be available from the authors.

* Corresponding author at: Department of Pediatric Surgery, Hospital for Children and Adolescents, University of Helsinki, PL 281, 00029, HUS, Helsinki, Finland. Tel.: + 35840 7241485.

E-mail address: malla.neuvonen@helsinki.fi (M. Neuvonen).
}

dysfunction including infertility and erectile difficulties in males [6-9]. EPT may carry less risk of these than other techniques because dissection of the distal rectum within the pelvis is carried out under direct vision on the rectal wall, and initially within a mucosal cuff in the Soavelike approach. The few uncontrolled studies available after EPT lend support to this notion $[10,11]$. There is very limited information concerning the outcomes for sexual function in adulthood in patients with HD $[7,12,13]$. This study has aimed to evaluate the long-term outcomes for LUTS and sexual function after EPT for HD in relation to ageand gender-matched peers from the general population, in order to provide further information on the safety of these procedures regarding preservation of the functional integrity of the genitourinary tract.

\section{Methods}

\subsection{Patients}

All patients aged $\geq 4$ years who underwent EPT at our center after histological verification of HD between 1987 and 2011 were identified from records. Patients with an intellectual disability due to an associated syndrome and patients with a definitive endostomy were excluded from the analysis. Patients (or their caregivers) were contacted twice 
by post between 2012 and 2013 and invited to answer detailed questionnaires concerning LUTS (all patients) and sexual function (patients aged $\geq 16$ years only). Voluntary participants were also invited to attend a clinical follow-up that included a clinical examination, urine flowmetry, renal tract ultrasound, and laboratory tests (infection parameters, electrolytes, creatinine, blood count, and urinalysis). An independent investigator who had not been involved in their surgical care contacted the patients. Operative details were obtained retrospectively from records. The ethics committee of the Helsinki University Hospital approved the research protocol.

\subsection{Questionnaires}

Of the nine items concerning LUTS (Appendix A), items three to nine were adapted from the previously validated Danish Prostatic Symptom Score [14]. Sexual function was enquired from patients aged $\geq 16$ years (Appendix B), including assessment of erectile function in males according to the previously validated Erectile Hardness Score (EHS) [15].

\subsection{Renal tract ultrasound}

Renal tract ultrasound including residual volume was performed by a pediatric radiologist blinded to the clinical outcomes.

\subsection{Urine flowmetry}

Urine flowmetry was performed using a spinning disc transducer URODYN® 1000 (@ Mediwatch Plc 2008). The flowmetry curves were classified as belly-shaped (normal) or tower,- plateau-, staccato- and interrupted-shaped (abnormal) [16]. A tower-shaped curve was defined as a high-amplitude curve of short duration. A plateau curve was a low amplitude and even curve. A staccato pattern was a fluctuating curve or curve with multiple peaks. An interrupted curve was defined as a curve reaching the baseline during voiding.

\subsection{Controls}

The controls were obtained from a reference pool of 594 Finnish subjects aged 4-26 years who had been randomly selected from the Population Register Centre of Finland and had answered identical questionnaires to patients [17]. From this pool, three controls matched for age and gender were randomly selected for each patient.

\subsection{Operative principles}

As shown in Table 1, all patients underwent either totally transanal EPT $(n=12)$ or EPT in combination with transabdominal mobilization of the colon $(n=44)$ outside the pelvis proximal to the peritoneal reflection. In all cases, dissection of the rectum within the pelvis up to the peritoneal reflection was carried out transanally. Transanal mucosectomy was commenced at approximately $5 \mathrm{~mm}$ proximal to the dentate line, proceeding to full-thickness dissection after $3-4 \mathrm{~cm}$ thereafter $[18,19]$. One patient with panintestinal aganglianosis underwent an end jejunostomy and colectomy [20]. The length of aganglionosis was defined by pathological examination of frozen sections. Patients were operated on by the same team of our pediatric consultant colorectal surgeons who also followed them up to adulthood.

\subsection{Statistics}

Unless otherwise stated, data are presented as frequencies or medians \pm interquartile range (IQR). Statistics were calculated using SPSS Version 21.0. Categorical variables were compared using Chisquare or Fisher's exact test, and continuous variables using MannWhitney $U$ test. $P<0.05$ was considered significant.
Table 1

Baseline characteristics of respondents with normal cognition $(n=59)$.

\begin{tabular}{lll}
\hline & $\begin{array}{l}\text { Survey of LUTS } \\
(n=59)\end{array}$ & $\begin{array}{l}\text { Survey of sexual } \\
\text { function }(n=24)\end{array}$ \\
\hline Median age (years) & $15(9-21)$ & $22(18-24)$ \\
Sex (M:F) & $43: 16(3: 1)$ & $16: 8(2: 1)$ \\
Family history of HD & $10(17)$ & $3(13)$ \\
Age at PT surgery (weeks) & $8(3-36)$ & $21(11-77)$ \\
Level of aganglionosis & & \\
Rectosigmoid & $51(86)$ & $22(92)$ \\
Long segment & $6(10)$ & $2(8)$ \\
Total colon & $2(3)$ & $0(0)$ \\
Preoperative decompressive enterostomy & $6(10)$ & $4(17)$ \\
Operation type & & \\
EPT* & $12(20)$ & $0(0)$ \\
EPT* with lapatoromy/laparoscopy & $44(75)$ & $23(96)$ \\
Ileoanal PT with a J-pouch & $3(5)$ & $1(4)$ \\
Re-do PT & $1(2)$ & $1(4)$ \\
ACE & $4(7)$ & $0(0)$ \\
Recurrent enterocolitis & $21(36)$ & $6(25)$ \\
Botox-injections & $7(12)$ & $1(4)$ \\
Myectomy & $4(7)$ & $2(8)$ \\
\hline
\end{tabular}

Data are frequencies (percentage) or medians (IQR).

EPT $=$ endorectal pull-through

$\mathrm{PT}=$ pull-through operation.

* includes procedures with simultaneous colon biopsies through umbilical incision, and procedures with a stoma closure in conjunction.

\section{Results}

\subsection{Patient cohort}

Of a total of 126 patients, three had died and 79 (64\%) returned completed questionnaires. Of these, 18 had an intellectual disability due to an associated syndrome and 2 had a definitive endostomy, leaving 59 eligible responses (median age 15 (IQR 9-21) years; 73\% male) for the assessment of LUTS, and 24 responses from patients $\geq 16$ years of age (median age 22 (IQR 18-24) years; 67\% male) for the assessment of sexual function and fertility. The baseline characteristics of respondents are summarized in Table 1 . The median age of controls $(n=177)$ for the assessment of LUTS was 14 (IQR 9-21; 73\% male) years and 22 (IQR $18-24)$ years for the survey on sexual function $(P=1.0$ for both vs. patients). Thirty-one out of 59 patients (53\%; median age 14 (IQR 9-22)) years also participated in the clinical follow-up.

\subsection{Drop-out analysis}

There was no significant difference in the main patient characteristics between respondents $(n=79)$ and non-respondents $(n=44)$ in terms of age, gender, and level of aganglionosis $(P=N S)$ making a significant selection bias unlikely.

\subsection{Lower urinary tract symptoms}

As shown in Fig. 1, the overall LUTS profiles of patients and controls were comparable for all symptoms apart from straining to void, which was reported by a higher proportion of controls than patients $(P=$ 0.008 ) In addition, controls tended to have more symptoms of urinary urgency than patients $(P=0.060)$. Overall, $67 \%$ of patients $(n=40$ / $59)$ and $80 \%$ of controls $(n=141 / 177)$ reported at least one type of LUTS $(P=0.062)$. However, most symptoms occurred only seldom in both; frequent (>1/week) LUTS were reported by $14 \%(n=8 / 59)$ of patients and $16 \%(n=28 / 177)$ of controls (Fig. $1 ; P=0.68)$ overall. The prevalence of any LUTS $>1$ /week was $8 \%$ in patients treated with totally transanal EPT ( $n=1 / 12)$, and $15 \%$ among patients with laparotomy- or laparoscopy-assisted EPT or IAA ( $n=7 / 47, P=1.0)$. Eighty-three per cent of patients ( $n=49 / 59)$ and $72 \%$ of controls $(n=128 / 177)$ voided 


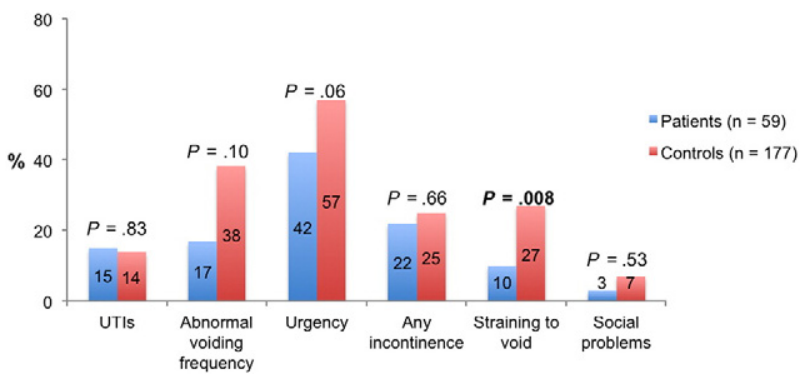

Fig. 1. Prevalence of any impairment in urinary function (\%). Patients with an intellectual disability or a definitive endostomy were excluded.

4-8 times per day $(P=0.099)$. No patient and $3 \%$ of controls $(n=6 /$ $177)$ had a voiding frequency $>8$ times per day $(P=0.34)$. Social problems due to LUTS were uncommon: $3 \%$ in patients $(n=2 / 59)$ and $7 \%$ in controls $(n=12 / 177 ; P=0.53)$.

\subsection{Clinical investigations}

The urine flowmetry results are shown in Table $2(n=29)$. Two patients refused urine flowmetry. The voided volume was $\geq 100 \mathrm{ml}$ in all patients. The flowmetry curve was normal belly-shaped in 15 (52\%) cases. Although the curve was plateau-shaped ( $n=4 / 29 ; 14 \%$ ), tower-shaped ( $n=2 / 29 ; 7 \%)$, staccato-shaped $(n=3 / 29,10 \%)$ or interrupted ( $n=5 / 29 ; 17 \%$ ) in the remaining 14 patients, there was no significant differences in the overall prevalence of any LUTS between patients with a belly-shaped curve $(67 \%(n=10 / 15))$ and an abnormal curve $(86 \%$ ( $n=12 / 14)$, nor in the prevalence of frequently occurring LUTS $(20 \%(n=3 / 15)$ vs. $21 \%(n=3 / 14)$, respectively; $P=$ NS for both). One 21 year-old patient with an interrupted curve and significant difficulties emptying the bladder was found to have a stricture of the bladder neck in the further urological investigations. This patient had undergone laparotomy-assisted EPT (2\% of all participants) without other complications during or after the procedure. The flowmetry findings were not associated with significant (frequent) LUTS, renal tract anomalies or abnormal residual volume in the remainder of cases.

\subsection{Renal tract ultrasound}

The renal tract ultrasound was normal in all 32 patients studied (median age 13 years), and all patients had normal residual urine volume of a median of 6 (IQR 3-12) ml, including the patient with a urethral stricture. Urinalysis, including urine cultures, were unremarkable. All blood and urine creatinine values were normal.

\subsection{Sexual function}

As shown in Fig. 2, the responses of patients and controls for items concerning sexual functional outcomes were not significantly different $(P>0.10)$, apart from a lower proportion of female patients that were currently in a stable relationship $(25 \% ; n=2 / 8$ vs. $83 \% ; n=20 / 24$,

Table 2

Urine flowmetry results for HD patients with normal cognition aged $\geq 4$ years $(n=29)$.

\begin{tabular}{llc}
\hline & Median & IQR \\
\hline Voiding time $(\mathrm{s})$ & 26 & $20-42$ \\
Time of flow (s) & 24 & $19-33$ \\
Max flow rate $(\mathrm{ml} / \mathrm{s})$ & 24 & $19-36$ \\
Time to max flow $(\mathrm{s})$ & 10 & $8-13$ \\
Flow rate $(\mathrm{ml} / \mathrm{s})$ & 14 & $10-23$ \\
Voided volume $(\mathrm{ml})$ & 411 & $287-553$ \\
Post-void residual $(\mathrm{ml})$ & 6 & $3-6$ \\
No. flow discontinuous & 2 & \\
\hline
\end{tabular}

$\mathrm{IQR}=$ interquartile range.
$P=0.005)$. There was no significant difference in relationship status between male patients $(40 \% ; n=6 / 16)$ or controls $(48 \% ; n=23 / 48 ; P=$ $0.59)$. All male patients $(n=16)$ and $94 \%$ of male controls $(n=44 / 48)$ reported completely normal erectile function (EHS $4 / 4 ; P=0.61$ ). All male patients and all controls reported their first ejaculations at the median age of 13 (IQR $12-14)$ years $(P=0.54)$. The age of coital debut was comparable between patients and controls; the median ages for male patients and controls were 16 (IQR 15-16) and 16 (IQR 15-18) years, $(P=0.53)$, and for female patients and controls 16 (IQR 16-18) and 16 (IQR $15-17)$ years $(P=0.42)$. Only three male patients had attempted to become parents; they each had one healthy child, and none reported any fertility issues or treatment.

\section{Discussion}

This cross-sectional study aimed to describe the long-term urinary and sexual functional outcomes among patients operated on for HD with EPT in relation to healthy age-matched controls. Our study suggests that the prevalence of LUTS is comparable to matched peers (Fig. 1), and sexual health including erectile function in males is preserved in patients treated with this approach (Fig. 2).

To date, follow-up studies of HD have mostly focused on bowel functional outcomes, which has been important as preservation of fecal continence and avoidance of functional complications are central issues in the management of these patients $[3,7,21,22]$. More recently, the prevalence of urological and sexual problems have also come under investigation due to the potential for these to result from pelvic dissection during surgery $[3,23,26]$. Most of the innervation to the bladder is closely related to the rectovesical pouch in males and the rectouterine pouch in females. As HD is not natively associated with congenital hypoplasia of the pelvic floor or spinal dysraphism as in certain other developmental disorders of the hindgut such as severe anorectal malformations and sacrococcygeal teratoma [24,25,32], significant LUTS and/or sexual dysfunction among these patients are likely to be the result of iatrogenic injuries from surgery. Pelvic dissection close to the rectal wall is the aim of all surgical approaches to HD. Also EPT carries a potential risk to other structures in the vicinity [12,26], although appropriate pelvic dissection within the wall of the distal rectum in EPT should prevent injury to the genitourinary tract.

Our study suggests that both patients and controls reported minor LUTS quite commonly and at comparable prevalence overall ( $64 \%$ of patients and $79 \%$ of controls; $P=\mathrm{NS}$ ), which is in accordance with the conclusions of Granström et al. after classic techniques for HD [21]. The higher prevalence of occasional (seldom) straining to void among controls ( $56 \%$ vs. $41 \%$ among patients) and occasional urgency ( $27 \%$ among controls vs. $12 \%$ in patients; $P \leq 0.046$ for both) is likely to reflect the sensitivity of our questionnaire for picking up minor symptoms and may be coincidental. Importantly, however, the results do not suggest that LUTS are any more common after EPT for HD than in controls. In our series, frequent LUTS of any kind ( $>1$ /week) also affected comparable proportions of patients (14\%) and controls $(16 \% ; P=0.068)$. These are similar to the $10-12 \%$ reported in previous uncontrolled series after Duhamel and Swenson procedures, where the symptom enquiry did not include an enquiry of symptom frequency [7]. A urethral stricture was identified in one patient following a laparotomy-assisted EPT (2\%), which was performed without complications, highlighting the possibility of iatrogenic injuries also after transanal mucosal dissection. The patient had no other long-term postoperative complications such as bowel dysfunction or enterocolitis. Laparoscopy- or laparotomy- assistance for colonic mobilization outside the pelvis should not, in theory, carry any greater risk of urologic injury than totally transanal EPT, as the pelvic component of the dissection is carried out transanally in all cases. Similarly, the intestino-anal anastomosis was performed using interrupted absorbable sutures for both IAA with J-pouch and coloanal anastomoses. In our series, patients with rectosigmoid disease combined laparoscopy/laparotomy-assisted approach was more commonly 

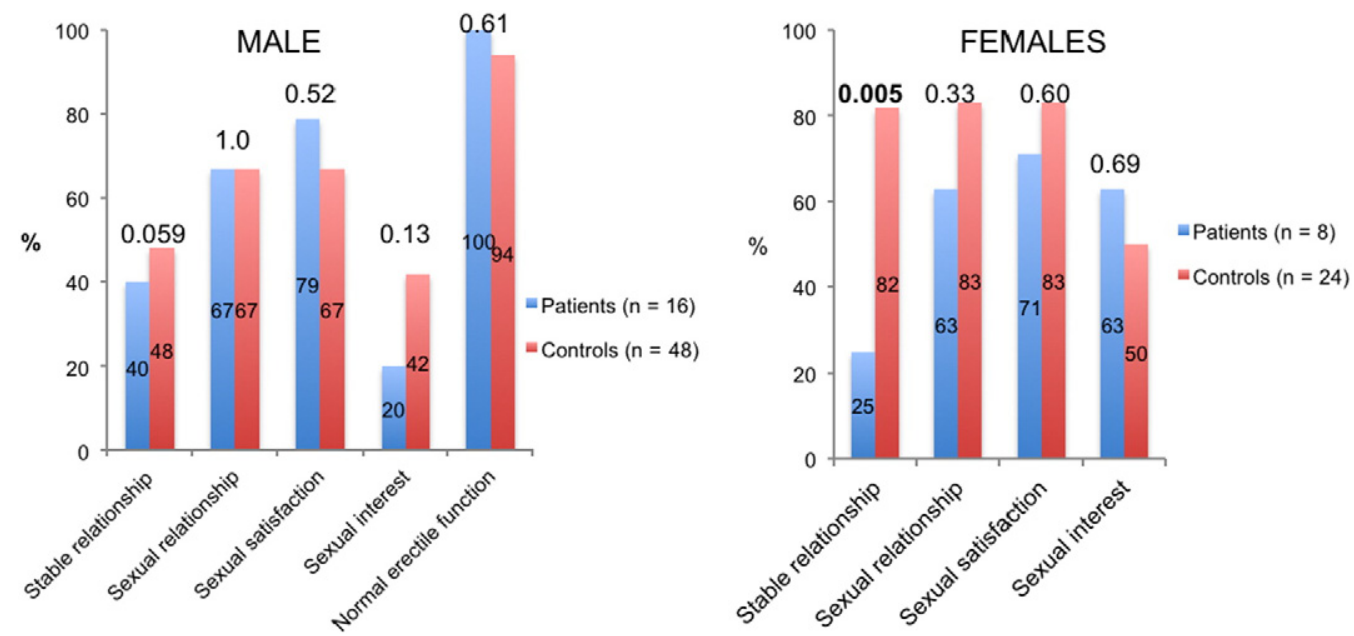

Fig. 2. Sexual function among respondents aged $\geq 16$ years.

employed than totally transanal EPT, because most patients were operated before we adopted totally transanal procedure in 2000. Recently, it has also been suggested that totally transanal techniques may damage the internal anal sphincter more than combined techniques due to prolonged intraoperative stretching [26].

Of the few studies on postoperative sexual problems among HD patients, Moore et al. [7] noted that $5 \%$ of patients reported sexual dysfunction including infertility and erectile dysfunction in males, especially after Duhamel and Swenson procedures. Van den Hondel et al. [12] showed in their recent uncontrolled study that $11 \%$ of the men with HD had severe erectile dysfunction but no ejaculation difficulties, and that $53 \%$ of the women with HD had sexual dysfunction and $20 \%$ sexual distress, which, however, were not associated with the length of the aganglionosis or type of operation. Our study did not identify any defects in physical sexual functioning after EPT compared to matched peers, supporting the safety of EPT with regard to preservation of such functions. However, insufficient numbers of patients had attempted to become parents to enable conclusive comments on fertility issues.

From a psychosocial perspective, patients with HD are exposed to factors that may negatively influence their sexual development. Anal dilatations and bowel management programs during childhood may lead to repetitive negative attention to genital area and potentially cause inhibitions with regard to sexual activities later in life [12]. Psychosocial comorbidity such as depression and anxiety resulting from deficient fecal control may hinder making close relationships [27-29]. Additionally, the embarrassment to report sexual problems may lead to underestimation of sexual dysfunction [12].

In our study, a lower proportion of female patients (25\%) than controls $(83 \%)$ were currently in a stable relationship $(P=0.005)$, but there was no such difference among males (Fig. 2). This could suggest some degree of psychosocial morbidity among female patients, although the numbers of females in this study is too small for definitive conclusions. The comparable age of coital debut between patients and controls in both genders nonetheless encouragingly suggests comparable willingness to commence intimate relationships. Among patients with severe ARMs in whom residual impairment of fecal control persists into adulthood in a significant proportion $[30,31]$, patients had a later coital debut, although comparable proportions were in stable relationships [30]. In these studies, female patients in particular reported a significant negative effect on sexual life from bowel functional issues, and fear of fecal incontinence has been strongly associated with perceived difficulties in forming close personal relationships in females but not in males [27]. Among our patients with HD, we have previously shown that fecal control becomes largely comparable to controls by adulthood [33,34], and therefore it is possible that bowel functional issues during childhood and puberty may continue to impact on the psychosexual well-being of female patients in particular in later life. In the study by Van den Hondel [12], over half of the patients with anorectal malformations and Hirschsprung's disease expressed receiving insufficient medical care regarding sexuality, and addressing these issues sensitively at an appropriate age during follow-up may be very important.

The limitations of this study include the relatively small number of patients, and larger multi-center studies are needed to confirm the validity of our findings. Studies comparing EPT and transanal Swensonlike EPT are also needed, as variations in preference for the type of procedure exist between centers. Although there was no significant difference between responders and non-respondents to our study in the drop-out analysis, the potential for selection bias is acknowledged. Although our questionnaires were detailed, they were not formally validated for this patient population.

\section{Conclusions}

Our current series supports the safety of EPT with regard to preservation of the integrity of the genitourinary and physical sexual function among HD patients.

\section{Appendix A. Lower Urinary Tract Symptoms Questionnaire}

1. Have you (Has your child)* had urinary organ diseases?

- Yes

- No

2. Have you (Has your child) had a urinary tract infection?

- Yes

- No

3. How many times do you (does your child) pass urine each day?

- 1-3 times

- 4-8 times

- More than 8 times

4. Do you (Does your child) need to strain to start/continue urination?

- No

- Seldom

- Often

- Always

5. Do you (Does your child) get a sudden urge to pass urine?

- No

- Seldom

- Often

- Always

" Questions phrased "your child" for parents of respondents less than 8 years of age. 
6. Is the urge so strong that urine escapes before reaching the toilet?

- No

- Seldom

- Often

- Always

7. Does urine ever leak upon straining (e.g. laughing, sneezing or coughing)?

- No

- Seldom

- Often

- Always

8. Does urine ever leak without physical activity or need to urinate?

- No

- Seldom

- Often

- Always

9. Do you (Does your child) have to strain to start/continue voiding?

- Never

- Seldom (less than once a week)

- Often (more than once a week)

- Always

10. Do you (Does your child) wet bed at night?

- Never

- Less than once a week

- More often than once a week

- Every night

11. Do you (Does your child) have to wake up at night to urinate?

- Never

- Once per night

- Twice per night

- Three times per night or more

12. Do you (Does your child) have social problems due to urinary incontinence?

- No

- Yes, due to daytime urinary incontinence only

- Yes, due to night-time urinary incontinence

- Yes, due to day- and night time urinary incontinence

13. How satisfied are you to your bladder function on a scale 1-5?

\section{Appendix B. Sexual Health Questionnaire}

1. How often do you feel sexual desire or interest?

- In most cases

- Sometimes

- Seldom

2. Are you in a regular relationship?

- Yes

- No

3. Have you been so far in a sexual relationship?

- No

- Yes

4. If you have had sexual activity, how often do you get satisfaction of it?

- No sexual activity

- Seldom

- Often

- Most of the times

\section{Females}

5. In what age did you have your first menstruation?

6. The length of menstruation (days)?

\section{Menstruation}

- Regular

- Irregular

- Currently using contraceptive pills: yes/no

Males

1. In what age did you have your first ejaculation?

2. Does your penis harden during the sexual excitement?

- Does not swell up nor stiffen

- Swells up but does not stiffen

- Stiffens but does not harden

- Stiffens and hardens

Both genders

3. Have you tried to have a child with your partner?

- No

- Yes, my spouse (men)/I (women) have not conceived yet

- Yes, my spouse (men)/I (women) have been conceived

- My spouse is (men)/I am (women) pregnant at the moment

4. Do you have children?

- No

- Yes, how many children?

- Yes, my spouse is (men)/I am (females) pregnant at the moment

5. Have you needed examinations or treatments for your fertility?

- I have not tried to get conceived yet

- There has been no need for it - pregnancies have succeeded

- No, but we are considering the option

- Yes

\section{References}

[1] Dasgupta R, Langer JC. Transanal pull-through for Hirschsprung disease. Semin Pediatr Surg 2005;14:64-71.

[2] Langer JC, Durrant AC, de la Torre L, et al. One-stage transanal soave pullthrough for Hirschsprung disease. Ann Surg 2003;238:569-83.

[3] De La Torre L, Langer JC. Transanal endorectal pull-through for Hirschsprung disease: technique, controversies, pearls, pitfalls, and an organized approach to the management of postoperative obstructive symptoms. Semin Pediatr Surg 2010;19:96-106.

[4] Shankar KR, Losty PD, Lamont GL, et al. Transanal endorectal coloanal surgery for Hirschsprung's disease: experience in two centers. J Pediatr Surg 2000;35:1209-13.

[5] Rintala RJ, Pakarinen MP. Long-term outcomes of Hirschprung's disease. Semin Pediatr Surg 2012;21:336-43.

[6] Boemers TM, Bax NMA, van Gool JD. The effect of rectosigmoidectomy and Duhameltype pull-through procedure on lower urinary tract function in children with Hirschsprung's disease. J Pediatr Surg 2001;36:453-6.

[7] Moore SW, Albertyn R, Cywes S. Clinical outcome and long-term quality of life after surgical correction of Hirschsprung's disease. J Pediatr Surg 1996;31:1496-502.

[8] Rescorla FJ, Morrison AM, Engles D, et al. Hirschsprung's disease. Evaluation of mortality and long-term function in 260 cases. Arch Surg 1992;127:934-41.

[9] Tariq GM, Brereton RJ, Wright VM. Complications of endorectal pull-through for Hirschsprung's disease. J Pediatr Surg 1991;26:1202-6.

[10] Ateş O, Hakgüder G, Kart Y, et al. The effect of dilated ganglionic segment on anorectal and urinary functions during 1-stage transanal endorectal pull through for Hirschsprung's disease. J Pediatr Surg 2007;42:1271-5.

[11] Kim AC, Langer JC, Pastor AC, et al. Endorectal pull-through for Hirschsprung's disease - a multicenter, long-term comparison of results: transanal vs transabdominal approach. J Pediatr Surg 2010;45:1213-20.

[12] Van den Hondel D, Sloots CE, Bolt JM, et al. Psychosexual well-being after childhood surgery for anorectal malformation or Hirschsprung's disease. J Sex Med 2015;12: $1616-25$.

[13] Ieiri S, Nakatsuji T, Akiyoshi J, et al. Long-term outcomes and the quality of life of HD in adolescent who have reached 18 years or older - a 47-year single-institute experience. J Pediatr Surg 2010;45:2398-402.

[14] Schou J, Poulsen AL, Nordling J. The value of a new symptom score (DAN-PSS) in diagnosing uro-dynamic infravesical obstruction in BPH. Scand J Urol Nephrol 1993; 27:489-92.

[15] Mulhall JP, Goldstein I, Bushmakin AG, et al. Validation of the erection hardness score. J Sex Med 2007;4:1626-34.

[16] Bauer SB, Nijman RJ, Drzewiecki BA, et al. International Children's continence society standardization report on urodynamic studies of the lower urinary tract in children. Neurourol Urodyn 2015;34:640-7.

[17] Kyrklund K, Taskinen S, Rintala RJ, et al. Lower urinary tract symptoms from childhood to adulthood: a population based study of 594 Finnish individuals $4-26$ years old. J Urol 2012;188:588-93. 
[18] Rintala RJ, Lindahl H. Transanal endorectal coloanal anastomosis for Hirschsprung's disease. Pediatr Surg Int 1993;8:128-31.

[19] Rintala RJ. Transanal coloanal pull-through with a short muscular cuff for classic Hirschsprung's disease. Eur J Pediatr Surg 2003;13:181-6.

[20] Hukkinen M, Koivusalo A, Rintala RJ, et al. Restorative proctocolectomy with J-pouch ileoanal anastomosis for total colonic aganglionosis among neonates and infants. J Pediatr Surg 2014;49:570-4.

[21] Granström AL, Danielson J, Husberg B, et al. Adult outcomes after surgery for Hirschsprung's disease: evaluation of bowel function and quality of life. J Pediatr Surg 2015;50:1865-9.

[22] Diseth TH, Bjørnland K, Novik TS, et al. Bowel function, mental health, and psychosocial function in adolescents with Hirschsprung's disease. Arch Dis Child 1997:76:100-6.

[23] Boemers TM. Urinary incontinence and vesicourethral dysfunction in pediatric surgical conditions. Semin Pediatr Surg 2002;11:91-9.

[24] Boemers TM, van Gool JD, de Jong TP, et al. Lower urinary tract dysfunction in children with bening sacrococcygeal teratoma. J Urol 1994;151:174-6.

[25] Boemers TM, Bax KM, Rövekamp MH, et al. The effect of posterior sagittal anorectoplasty and its variants on lower urinary tract function in children with anorectal malformation. J Urol 1995;153:191-3.

[26] Stensrud K, Emblem R, Bjørnland K. Anal endosonography and bowel function in patients undergoing different types of endorectal pull-through procedures for Hirschsprung disease. J Pediatr Surg 2015;50:1341-6.

[27] Grano C, Bucci S, Aminoff D, et al. Feelings of depression in people with ARM: the role of critical incidents and perceived difficulties in close and personal relationships. Pediatr Surg Int 2014;30:823-8.
[28] Bel LG, Vollebregt AM, Van der Meulen-de Jong AE, et al. Sexual dysfunctions in men and women with inflammatory bowel disease: the influence of IBDrelated factors and depression on sexual function. J Sex Med 2015;12: 1557-67.

[29] Kalmbach DA, Pillai V, Kingsberg SA, et al. The transaction between depression and anxiety symptoms and sexual functioning: a prospective study of premenopausal, healthy women. Arch Sex Behav 2015;44:1635-49.

[30] Kyrklund K, Pakarinen MP, Koivusalo A, et al. Long-term bowel functional outcomes in rectourethral fistulas treated with PSARP: controlled results after 4-29 years of follow-up: a single-institution, cross-sectional study. J Pediatr Surg 2014;49: $1635-42$.

[31] Kyrklund K, Pakarinen MP, Koivusalo A, et al. Bowel functional outcomes in females with perineal or vestibular fistula treated with anterior sagittal anorectoplasty: controlled results into adulthood. Dis Colon Rectum 2015;58: 97-103.

[32] Kyrklund K, Taskinen S, Rintala RJ, et al. Sexual function, fertility and quality of life after modern treatment of anorectal malformation. J Urol 2016 Available at: 10. 1016/j.juro.2016.08.079. [Epub ahead of print].

[33] Neuvonen MI, Kyrklund K, Lindahl HG, et al. A population-based, complete followup of 146 consecutive patients after transanal mucosectomy for Hirschsprung disease. J Pediatr Surg 2015;50:1653-8.

[34] Neuvonen MI, Kyrklund K, Rintala RJ, et al. Bowel function and quality of life after transanal pull-through for Hirschsprung's disease: controlled outcomes up to adulthood. Ann Surg 2016 [Epub ahead of print, Available at:] 10.1097/ SLA0000000000001695. 